\title{
Erratum to: Super-ensemble of three RCMs for climate projection over East Asia and Taiwan
}

\author{
Chung-Ming Liu • Ming-Chin Wu • Sahana Paul • Ying-Chen Chen • Shu-Hua Lin • \\ Wei-Shiang Lin • Yen-Chih Lee • Huang-Hsung Hsu • Ren-Yow Tzeng • \\ Cheng-Ta Chen
}

Published online: 11 May 2010

(C) Springer-Verlag 2010

\section{Erratum to: Theor Appl Climatol \\ DOI 10.1007/s00704-010-0275-x}

The original version of this article unfortunately contained a mistake. The spelling of Ren-Yow Tzeng's name was incorrect.

The online version of the original article can be found under doi:10.1007/s00704-010-0275-x.

C.-M. Liu • S. Paul • Y.-C. Chen · S.-H. Lin · W.-S. Lin • Y.-C. Lee Global Change Research Center, National Taiwan University,

Taipei, Taiwan, China

C.-M. Liu $(\square) \cdot$ M.-C. Wu $\cdot$ H.-H. Hsu

Department of Atmospheric Sciences,

National Taiwan University,

Taipei, Taiwan, China

e-mail: liucm@ntu.edu.tw

R.-Y. Tzeng

Department of Atmospheric Sciences,

National Central University,

Chungli, Taiwan, China

\section{C.-T. Chen}

Department of Earth Sciences, National Normal University,

Taipei, Taiwan, China 